\title{
A Recursive Digital Predistorter for Linearizing RF Power Amplifiers with Memory Effects
}

\author{
P. L. Gilabert ${ }^{1}$, G. Montoro ${ }^{1}$ and A. Cesari ${ }^{2}$ \\ ${ }^{1}$ Signal Theory and Communications Dpt., Universitat Politècnica de Catalunya, Barcelona, Spain. \\ ${ }^{2}$ Intégration de Systèmes de Gestion de 1'Energie, LAAS-CNRS, Toulouse, France. \\ E-mail: plgilabert@tsc.upc.edu
}

\begin{abstract}
A nonlinear auto-regressive moving average (ARMA) structure capable of compensating nonlinear memory effects in RF power amplifiers is here presented. Results on the linearity improvement, in both in-band and out-of-band distortion compensation, achieved by this baseband digital predistorter are provided. Moreover, a study on this nonlinear ARMA system stability is also reported.
\end{abstract}

Index Terms - Linearization of $R F$ power amplifiers, predistortion.

\section{INTRODUCTION}

Modern multilevel and multicarrier modulation formats present high peak to average power ratios (PAPR), what implies that for having linear amplification, significant back-off levels are required, thus penalizing PA's power efficiency. In addition, those efficient modulation formats (presenting high PAPRs) are also very sensitive to the inter-modulation distortion (IMD) that results from nonlinearities in the RF transmitter chain. Moreover coping with high speed envelope signals (presenting significant bandwidths) makes engineers reconsider the degradation suffered from memory effects. Therefore the use of linearizers to deal with the classic trade-off between linearity and efficiency is still a relevant issue to investigate [1].

Among linearizers, digital predistortion (PD) has been object of multiple publications in recent past years [2]. When comparing to other linearization methods, digital PD not only avoids the complexity of the RF hardware (every time operating at higher frequencies) but also is a huge field of research for engineers for optimizing DSP architectures and algorithms. Digital PD linearization is currently carried out by means of a high speed digital signal processors (DSP's, FPGA's), already present in most of current communication equipment for mandatory issues within the wireless standards.

The cancellation performance of digital predistorters is sensitive to memory effects generated in RF power amplifiers. Digital PD based on memoryless models that only take into account the $\mathrm{AM} / \mathrm{AM}$ and AM/PM static curves achieve acceptable results when dealing with narrowband signals (e.g. nearly-constant envelope modulations). However, modern communications systems are aimed at increasing capacity and spectral efficiency by using spread spectrum (e.g. WCDMA) or multicarrier (e.g. OFDM) techniques combined with multilevel modulation formats (e.g. M-QAM) along moderate channel bandwidths, so memory effects in the PA can no longer be ignored.

\section{Modeling AND CANCELING MEMORY EFFECTS IN RF POWER AMPLIFIERS}

The most common memory effects recognized in literature are due to electrical and thermal dispersion effects [3]. So then, taking into account those effects, the PA output amplitude and phase not only depend on the instantaneous input amplitude, but also on their past values. Some dynamic nonlinear models considered in literature for identifying and compensating PA's nonlinear behavior considering memory effects ([2],[3]) are: discrete Volterra series; Wiener-Hammerstein models (or both combined) and their variations (parallel-cascade configurations) and Neural Networks. Several of this configurations have been analyzed an applied in [4] and [5].

\section{GENERAL FORMULATION OF THE PROPOSED NONLINEAR ARMA SYSTEM}

Our goal is to develop a nonlinear auto-regressive moving average (ARMA) structure inherently stable despite the IIR term. Soury et al. in [6] point out that in systems where memory effects are secondary effects to a static nonlinear behavior, it is possible to consider that the individual signal pulses propagate nonlinearly in time but tend to sum up linearly. Hence, PA's memory effects due to biasing networks and thermal effects will fall in this category. A simplified version of the discrete-time Volterra kernels, considering a static nonlinear behavior dominant in front of memory effects, is described as:

$$
y(n)=\sum_{i=0}^{M} f_{i}(x(n-i))
$$

where $f_{i}($.$) is a nonlinear function (\mathrm{i}=1,2 \ldots \mathrm{M})$, and the present output sample depends on the sum of 
different static nonlinearities, considering present and past samples of the input. The signals $x(n)$ and $y(n)$ represent the complex envelope (low-pass equivalent) of the PA input and output, respectively [6].

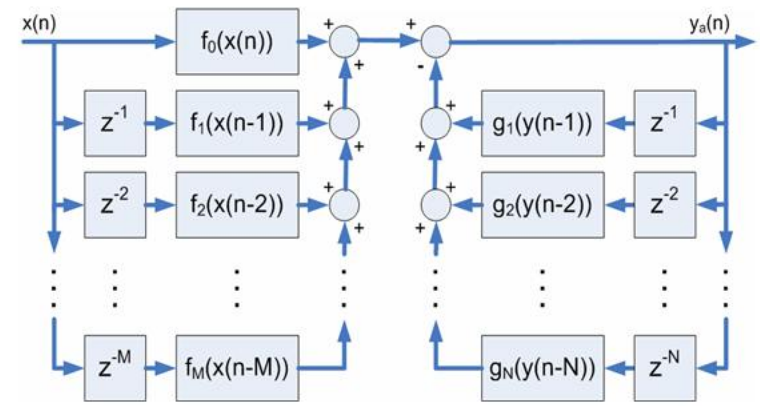

Fig. 1. Proposed nonlinear ARMA system.

\section{A. Formulation of the Proposed Nonlinear ARMA System for Modeling and Predistorting}

By considering a more general formulation than (1), we have taken into account both PA's input and output delays. So then, the general expression for our proposed nonlinear ARMA model is:

$$
y(n)=\sum_{i=0}^{M} f_{i}(x(n-i))-\sum_{i=1}^{N} g_{i}(y(n-i))
$$

This kind of structure can take to instabilities due to the nonlinear $\left(\mathrm{g}_{\mathrm{i}}\right)$ IIR terms. Therefore, in order to use it for PD, a prior stability test will be necessarily carried out.

B. Stability test: Small-Gain Theorem. Application to the Proposed Nonlinear ARMA System

The known small-gain theorem is an input-output stability method based in bounded norms, capable of determining the stability in nonlinear systems. It was developed by G. Zames in 1966 and is well explained and applied in [7].

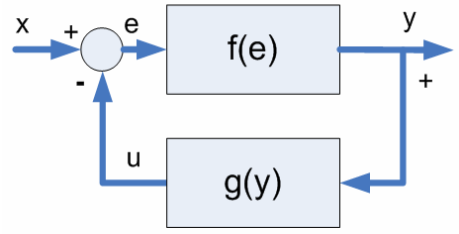

Fig. 2. Block interconnections for applying the small gain stability method.

From Fig. 2 it follows the inequality (3). That means: the p-norm of the system output is bounded and that bound exists if $\gamma\{f\} \cdot \gamma\{g\}<1$. This is a sufficient stability condition [7].

$$
\|y\|_{p} \leq \frac{\gamma\{f\}}{1-\gamma\{f\} \gamma\{g\}}\|x\|_{p}
$$

In our case, we define $\|.\|_{2}$ as the second order norm, and being $\gamma\left\{f_{i}\right\}$ and $\gamma\left\{g_{i}\right\}$ bounds for the second order norm gain of each of the polynomial terms $f_{i}(\cdot)$,

$g_{i}(\cdot)$. Then it follows:

$$
\|y\|_{2}=\sum_{i=0}^{M} \gamma\left\{f_{i}\right\}\|x\|_{2}+\sum_{i=1}^{N} \gamma\left\{g_{i}\right\}\|y\|_{2}
$$

and finally we obtain this input-output inequality:

$$
\|y\|_{2}<\frac{\sum_{i=1}^{M} \gamma\left\{f_{i}\right\}}{1-\sum_{i=1}^{N} \gamma\left\{g_{i}\right\}}\|x\|_{2}
$$

So, the stability is assured (a sufficient condition) if the inequality $\sum_{i=1}^{N} \gamma\left\{g_{i}\right\}<1$ is accomplished [7].

\section{Proposed Nonlinear ARMA Predistorter}

In order to train the nonlinear ARMA based digital predistorter, an indirect learning approach or translation method has been considered. In the indirect learning, a postdistorter is first estimated by using the power amplifier input and output baseband modulated data.

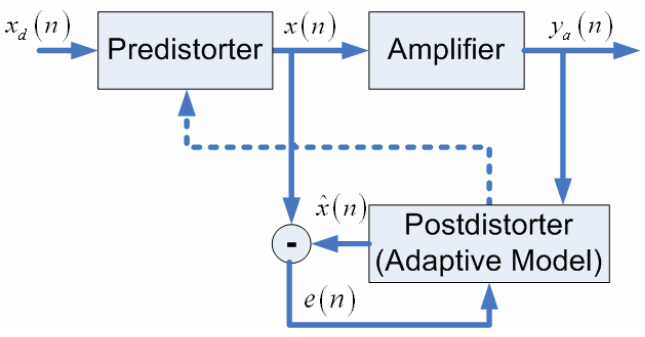

Fig. 3. Indirect learning approach for training a digital PD.

Later, the already estimated postdistorter coefficients are copied to an identical model that is used to predistort the modulated input signal that will be fed to the PA (see Fig. 3).

\section{A. Nonlinear ARMA Predistorter Formulation}

Let us consider the general expression in (2), and use this nonlinear ARMA formulation to estimate the proposed postdistorter. To do that, we have considered 
general power series to model nonlinear functions $\left(f_{i}(\cdot), g_{i}(\cdot)\right)$, as:

$$
\begin{aligned}
& \hat{x}(n)=\sum_{n=0}^{N} \beta_{n}\left(\sum_{p=0}^{P-1} \gamma_{p} \cdot y_{a}\left(n-\tau_{n}\right) \cdot\left|y_{a}\left(n-\tau_{n}\right)\right|^{p}\right)- \\
& -\sum_{m=1}^{M} \alpha_{m}\left(\sum_{p=0}^{P-1} \lambda_{p} \cdot x\left(n-\tau_{m}\right) \cdot\left|x\left(n-\tau_{m}\right)\right|^{p}\right)
\end{aligned}
$$

Being $x(n)$ and $y(n)$ the baseband input and output power amplifier modulated signals respectively. Where $P$ is the order of the memoryless polynomial with complex coefficients $\gamma_{p}$ and $\lambda_{p}, N$ and $M$ the number of the delayed input and output samples considered for modeling its dynamics and $\beta_{n}$ and $\alpha_{m}$ the complex coefficients of an IIR filter. In addition, $\tau_{n}$ and $\tau_{m}$ past samples are chosen in order to be the most significant delays contributing in the postdistorter estimation [5]. To identify the nonlinear ARMA coefficients of the postdistorter a quadratic cost function is defined and the nonlinear ARMA coefficients are estimated by means of a Fast-Kalman algorithm [5].

\section{B. Generation of Look-up Tables for the Nonlinear ARMA Predistorter}

Once the postdistorter coefficients are estimated, the predistorter has to be implemented. In order to implement the nonlinear ARMA model predistorter into a DSP/FPGA processor it is possible to rewrite (6) into a more general expression similar to (2).

$$
\begin{aligned}
& x(n)=\underbrace{d_{01} \cdot x_{d}(n)+\cdot \cdot+d_{0(P-1)} \cdot x_{d}(n)\left|x_{d}(n)\right|^{P-1}}_{L U T_{-} x_{d-} 0}+ \\
& \underbrace{d_{11} \cdot x_{d}\left(n-\tau_{1}\right)+\cdot \cdot+d_{1(P-1)} \cdot x_{d}\left(n-\tau_{1}\right)\left|x_{d}\left(n-\tau_{1}\right)\right|^{P-1}}_{L U T_{-} x_{d} 1}+ \\
& \vdots \\
& \underbrace{d_{N 1} \cdot x_{d}\left(n-\tau_{N}\right)+\cdot \cdot+d_{N(P-1)} \cdot x_{d}\left(n-\tau_{N}\right)\left|x_{d}\left(n-\tau_{N}\right)\right|^{P-1}+}_{L U T_{-} x_{-} N}(7) \\
& \underbrace{h_{11} \cdot x\left(n-\tau_{1}\right)+\cdot \cdot+h_{1(P-1)} \cdot x\left(n-\tau_{1}\right)\left|x\left(n-\tau_{1}\right)\right|^{P-1}+}_{L U T_{-} x_{-} M}+ \\
& \vdots \\
& \underbrace{}_{h_{M 1} \cdot x\left(n-\tau_{M}\right)+\cdot \cdot+h_{M(P-1)} \cdot x\left(n-\tau_{M}\right)\left|x\left(n-\tau_{M}\right)\right|^{P-1}}
\end{aligned}
$$

Being $x_{d}(n)$ and $x(n)$ the baseband modulated input data and the predistorted output data respectively, and where for simplicity $d_{n p}=\beta_{\mathrm{n}} \cdot \gamma_{\mathrm{p}}$ and $h_{m p}=\alpha_{\mathrm{m}} \cdot \lambda_{\mathrm{p}}$. Therefore this nonlinear ARMA model can be implemented as a scalable set of LUTs, as it is shown in Fig. 4.

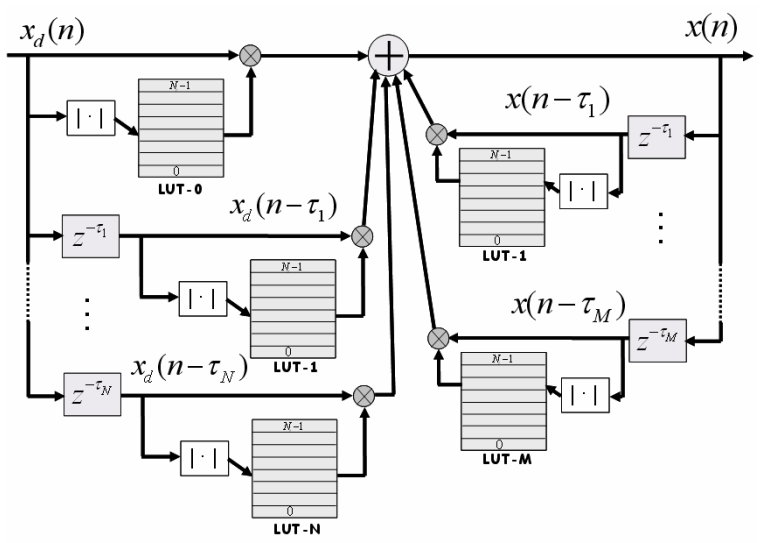

Fig. 4. Nonlinear ARMA multi-LUT implementation.

\section{Multi-Lut IMPLEMENTATION}

The baseband predistorter presented in this paper is well suited for implementation in FPGA's, because it is stated in terms of a basic predistortion cell (BPC) requiring simple hardware operations: LUT memory accesses, indexing and complex product computations, and a programmable shift register. The predistorter can be easily scaled-up as a function of the number of considered coefficients. In this way linearity performances can be increased without sacrifice the transmission bandwidth, by parallelization of as many BPC as necessary. Moreover, as it is shown in [5], complexity can be traded-off against linearity, by choosing the most relevant delays, therefore scalingdown the number of BPC involved.

A DSP external to the FPGA can perform the adaptive algorithm functions (operating in a different time-scale than real-time) and, by parameter passing, dynamically update the LUT contents for a given delay configuration. Furthermore, according to standard specifications or other criteria, the DSP can dynamically scale the delay configuration by switching on/off a number of BPC and reprogramming its shift register, in order to balance linearity and complexity in a multi-terminal or SDR scenario, for instance.

\section{RESULTS}

In order to estimate the predistorter a 16-QAM RRC filtered (roll-off 0.25 ) modulated test signal has been chosen. The RF signal bandwidth is $1.25 \mathrm{MHz}$, at a centre frequency of $1.96 \mathrm{GHz}$ corresponding to a WCDMA channel. The PA used is a LDMOS 3-stage class $\mathrm{AB}$ main amplifier operating at $2 \mathrm{~dB}$ of input back-off (IBO). For simplicity, all modulated data have been normalized. The nonlinear ARMA predistorter used consists in power series up to ninth 
order to model nonlinearities and an IIR filter with 3 taps, one zero $(\mathrm{N}=2$, because of the current sample) and two poles $(\mathrm{M}=2)$. First, let us check the stability criteria described in eq.(5). Fig. 5 shows how the sum of the estimated nonlinear functions modulus $g_{1}(\cdot), g_{2}(\cdot)$ are clearly below the bound (dashed line), assuring then, that this nonlinear ARMA PD will be inherently stable.

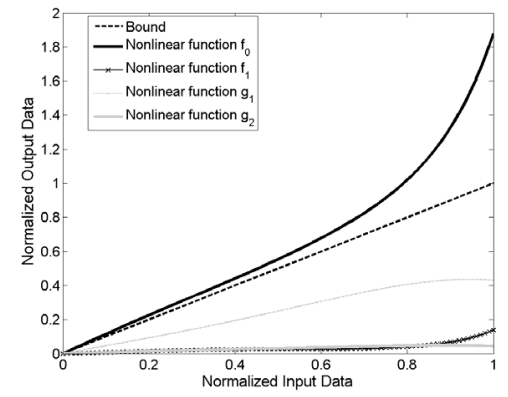

Fig. 5. Nonlinear functions of the nonlinear ARMA model.

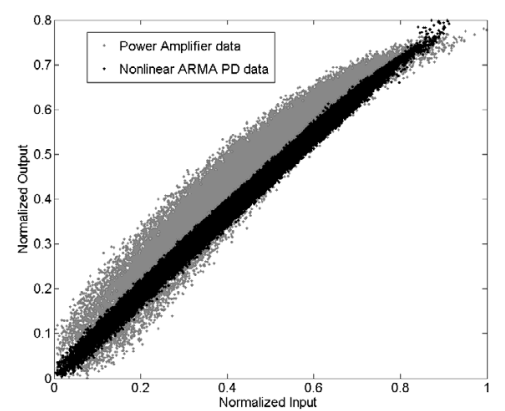

Fig. 6. Power amplifier and predistorted AM/AM curves.

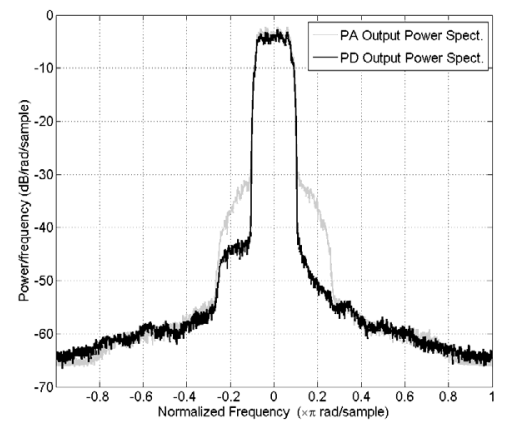

Fig. 7. PA and PD power spectra.

The nonlinear ARMA predistorter has been implemented with look-up tables, two LUT's for the input data (current sample and the delayed sample, $\left.f_{0}(\cdot), f_{1}(\cdot)\right)$ and another two LUT's for the delayed samples of the output data $g_{1}(\cdot), g_{2}(\cdot)$. Fig. 6 shows the AM/AM power amplifier and predistorted curves. It can be seen how the predistorted data looks more linear near compression (out-of-band compensation), at the time that presents less dispersion (in-band compensation). Fig. 7 shows at least $-10 \mathrm{~dB}$ of adjacent channel power ratio (ACPR) reduction. In addition, because of memory effects, this reduction is asymmetric, achieving up to $-20 \mathrm{~dB}$ ACPR reduction in the upper sideband. Fig. 8 shows the nonlinear ARMA predistorter in-band compensation by reducing the PA constellation blur.

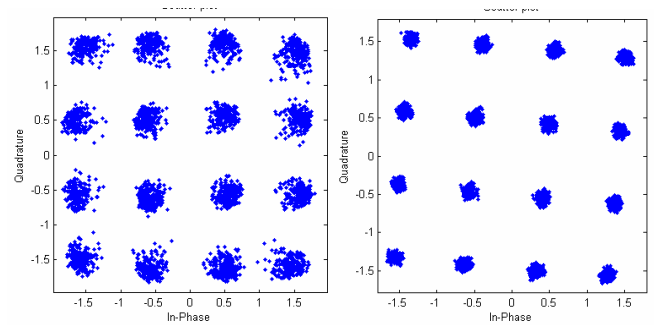

Fig. 8. Left: PA 16-QAM constellation, Right: Nonlinear ARMA PD 16-QAM constellation.

\section{CONCLUSION}

By using the proposed methodology it is possible to obtain nonlinear ARMA models inherently stables, useful for identifying and predistorting low pass complex envelope dynamic PA models. The ARMA structure here presented has shown better accuracy than more simplified Hammerstein and Wiener models and much better performance than memoryless models.

\section{ACKNOWLEDGMENT}

This work was partially supported by Spanish Government under project TEC2005-07985-C03-02, and by the EU network TARGET "Top Amplifier Research Group in a European Team" (IST-1507893-NOE). We also want to thank D. Silveira (Tech. Univ. of Wien) for his PA measurements.

\section{REFERENCES}

[1] Petteri Alinikula, "Multiradio Yelds Challenges for Mobile Phones," Microwave Journal, vol. 48, n 7, pp. 22-30, July 2005.

[2] W. J. Kim, S.P. Stapleton, J.H. Kim and C. Edelman, "Digital Predistortion Linearizes Wireless Power Amplifiers," IEEE Microwave Magazine, pp.54-61, September 2005.

[3] J. Vuolevi, "Distortion in RF Power Amplifiers", Artech House INC, 2003.

[4] P. L. Gilabert, G. Montoro and E. Bertran, "On the Wiener and Hammerstein Models for Power Amplifier Predistortion", APMC-05, vol. 2, pp.1191-1194, 4-7 December, Suzhou, China, 2005.

[5] P. L. Gilabert, G. Montoro and E. Bertran, "A Methodology to Model and Predistort Short-Term Memory Nonlinearities in Power Amplifiers", Proceedings of the INMMiC 2006. Aveiro, Portugal. January 2006.

[6] A. Soury, E. Ngoya, J. M. Nebus, “A New Behavioral Model taking into Account Nonlinear Memory Effects and Transient Behaviors in Wideband SSPAs", IEEE MTT Symp., pp. 853856, Seattle, USA, June 2002.

[7] C.A. Desoer and M. Vidyasagar, "Feedback Systems: InputOutput Properties”, Academic Press, New-York, 1975. 